\title{
Stereotactic radiosurgery combined with nivolumab or Ipilimumab for patients with melanoma brain metastases: evaluation of brain control and toxicity
}

Giuseppe Minniti ${ }^{{ }^{*}}$ (D), Dimitri Anzellini ${ }^{2}$, Chiara Reverberi ${ }^{2}$, Gian Carlo Antonini Cappellini ${ }^{3}$, Luca Marchetti ${ }^{1}$, Federico Bianciardi ${ }^{1}$, Alessandro Bozzao ${ }^{4}$, Mattia Osti ${ }^{2}$, Pier Carlo Gentile ${ }^{1}$ and Vincenzo Esposito ${ }^{5}$

\begin{abstract}
Purpose: To investigate the efficacy and safety of concurrent stereotactic radiosurgery (SRS) and ipilimumab or nivolumab in patients with untreated melanoma brain metastases.

Patients and Methods: Eighty consecutive patients with 326 melanoma brain metastases receiving SRS in combination with ipilimumab or nivolumab were identified from an institutional database and retrospectively evaluated. Patients started systemic treatment with intravenous nivolumab or ipilimumab within one week of receiving SRS. Nivolumab was given at doses of $3 \mathrm{mg} / \mathrm{kg}$ every two weeks. Ipilimumab was administered up to four doses of $10 \mathrm{mg} / \mathrm{kg}$, one every 3 weeks, then patients had a maintenance dose of $10 \mathrm{mg} / \mathrm{kg}$ every 12 weeks, until disease progression or inacceptable toxicity. Primary endpoint of the study was intracranial progression-free survival (PFS). Secondary endpoints were extracranial PFS, overall survival (OS), and neurological toxicity.

Results: Eighty patients were analyzed. Forty-five patients received SRS and ipilimumab, and 35 patients received SRS and nivolumab. With a median follow-up of 15 months, the 6-month and 12-month intracranial PFS rates were $69 \%(95 \% \mathrm{Cl}, 54-87 \%)$ and $42 \%(95 \% \mathrm{Cl}, 24-65 \%)$ for patients receiving SRS and nivolumab and $48 \%(95 \% \mathrm{Cl}, 34-64 \%)$ and $17 \%(95 \% \mathrm{Cl}, 5-31 \%)$ for those treated with SRS and ipilimumab $(p=0.02)$, respectively. Extracranial PFS and OS were 37 and 78\% in SRS and nivolumab group, respectively, and 17 and 68\% in SRS and ipilimumab group, respectively, at 12 months. Sub-group analysis showed significantly better intracranial PFS for patients receiving multi-fraction SRS ( $3 \times 9 \mathrm{~Gy})$ compared to single-fraction SRS (70\% versus $46 \%$ at 6 months, $p=0.01$ ), especially in combination with nivolumab. Grade 3 treatment-related adverse events occurred in 11 (24\%) patients treated with SRS and ipilimumab and 6 (17\%) patients who received SRS and nivolumab. Radiation-induced brain necrosis (RN) occurred in $15 \%$ of patients.
\end{abstract}

Conclusions: Concurrent SRS and ipilimumab or nivolumab show meaningful intracranial activity in patients with either asymptomatic and symptomatic melanoma brain metastases, although a subset of patients may develop symptomatic RN. The combination of nivolumab with SRS is associated with better intracranial control.

Keywords: stereotactic radiosurgery, melanoma brain metastases, fractionated stereotactic radiosurgery, checkpoint inhibitors, immunotherapy

\footnotetext{
*Correspondence: minnitig@upmc.edu

'Radiation Oncology Unit, UPMC Hillman Cancer Center|, San Pietro Hospital

FBF, 00189 Rome, Italy

Full list of author information is available at the end of the article
}

(c) The Author(s). 2019 Open Access This article is distributed under the terms of the Creative Commons Attribution 4.0 International License (http://creativecommons.org/licenses/by/4.0/), which permits unrestricted use, distribution, and reproduction in any medium, provided you give appropriate credit to the original author(s) and the source, provide a link to the Creative Commons license, and indicate if changes were made. The Creative Commons Public Domain Dedication waiver (http://creativecommons.org/publicdomain/zero/1.0/) applies to the data made available in this article, unless otherwise stated. 


\section{Introduction}

Brain metastases are a common and devastating complication of cancer affecting $25 \%$ of patients with advanced melanoma [1]; for these patients, systemic therapy and local treatments, including surgical resection, whole brain radiation therapy (WBRT) and stereotactic radiosurgery (SRS) have been the most common therapeutic options.

Systemic chemotherapy has been widely used in the past for patients with melanoma brain metastases, although it has shown a limited activity. Local control (LC) has improved with the use of surgery and radiotherapy, given alone or in combination [1]. Historically, WBRT has been the cornerstone for treatment of multiple brain metastases, but its use has been progressively replaced by SRS; its efficacy in patients with a limited number of lesions, usually 1-4, has been demonstrated in randomized trials [2, 3], although LC in melanoma patients is inferior than that reported for other histologies, especially when considering large tumors [4-7].

In the last few years systemic therapies have evolved; targeted therapies with BRAF and MEK inhibitors and immunotherapy with PD-1/PD-L1 or CTLA-4 checkpoint inhibitors, given alone or in combination, have significantly improved survival in patients with melanoma brain metastases [8-12]. In a randomized phase 2 study of 60 patients with melanoma brain metastases receiving combined nivolumab and ipilimumab or nivolumab alone, Long et al. [11] showed an intracranial response of $46 \%$ and $20 \%$, respectively; with a median follow-up of 17 months, 6-month intracranial progression-free survival (PFS) and overall survival (OS) rates were $35 \%$ and $68 \%$ in patients receiving nivolumab, and $53 \%$ and $78 \%$ in those receiving nivolumab and ipilimumab. In another phase 2 study of 94 patients with melanoma brain metastases treated with combined nivolumab and ipilimumab, Tawbi et al. [12] observed an intracranial objective response of 55\% lasting at least 6 months, with PFS rates of $70.4 \%$ and OS rates of $59.5 \%$ at 9 months.

In patients with melanoma brain metastases, stereotactic radiosurgery SRS in combination with checkpoint inhibitors may be associated with improved efficacy over SRS alone [13]; however, timing and sequence of combined SRS and checkpoint inhibitors are highly variable among different studies, and the efficacy and toxicity of treatment remains to be defined. In our study we have evaluated the efficacy and safety of SRS combined with nivolumab or ipilimumab in patients with untreated melanoma brain metastases.

\section{Patients and Methods}

Between September 2012 and December 2017, 112 consecutive patients $\geq 18$ years old receiving combined SRS and ipilimumab or nivolumab for one to ten melanoma brain metastases were retrospectively evaluated. In general, patients with lesions up to $2.5 \mathrm{~cm}$ in size were treated with single-fraction SRS, while larger lesions located near or in eloquent areas (i.e., motor, somatosensory, speech, visual cortices, basal ganglia, thalamus, and the brainstem) received multi-fraction SRS to minimize potential increased risk of late radiation-induced brain necrosis (RN).

All radiographic, surgical, and pathological information were drawn from a prospectively maintained database of patients with brain tumors treated at Sant' Andrea Hospital and UPMC Hillman Cancer Center San Pietro Hospital. Thirty-two patients were excluded due to insufficient clinical information, previous use of anti-PD-1/PD-L1, brain surgery or radiation. Previous adjuvant therapies, including ipilimumab or BRAF/MEK inhibitors, were allowed. A total of 80 patients with 326 brain metastases were finally analyzed. All patients provided written consent to the treatment. Local Institutional Review Boards at Sant' Andrea and San Pietro Hospitals approved this retrospective study.

All lesions were treated with LINAC-based SRS (TrueBeam STx and Clinac 2100 linear accelerators, Varian Medical System) using a commercial stereotactic mask fixation system (BrainLab). Target volumes were contoured on thin-slice (1-mm) gadolinium-enhanced T1-weighted axial MRI sequences fused with planning computed tomography (CT) scans. The gross tumor volume (GTV) was delineated as the contrast-enhancing tumor demonstrated on MRI scans. The planning tumor volume (PTV) was generated giving a geometric expansion to GTV of $0.5-1 \mathrm{~mm}$. In patients undergoing single-fraction SRS, doses were 22 Gy for lesions $<2 \mathrm{~cm}$ and 18 Gy for those between 2 and $3 \mathrm{~cm}$ in size. For lesions treated with multi-fraction SRS, a dose of $27 \mathrm{~Gy}$ in 3 fractions was delivered on consecutive days. The choice of 3x9 Gy was made on the basis of radiobiological consideration and previous clinical experiences $[14,15]$. According to the linear quadratic model for the estimation of dose-effect relationship adjusted for high doses [14], the biological effective dose (BED) of 27 Gy in 3 fractions is 40 Gy that corresponds to a single dose of about $22 \mathrm{~Gy}$, assuming an $\alpha / \beta$ of 12 Gy $\left(B_{12}\right)$ for brain metastases. Doses were generally prescribed to the $80 \%$ isodose line and delivered using 4-7 noncoplanar dynamic or volumetric arcs. Cone-beam CT and ExacTrac ${ }^{\ominus}$ image-guided systems were used to ensure accurate patient positioning. In patients with significant or symptomatic perilesional edema, a maximum dose of $4 \mathrm{mg}$ dexamethasone per day was allowed at the time of SRS, then maintained for 3-7 day.

Concurrent systemic treatment consisted of - intravenous nivolumab administered at doses of $3 \mathrm{mg} / \mathrm{kg}$ every 
two weeks, or - intravenous ipilimumab up to four doses of $10 \mathrm{mg} / \mathrm{kg}$, one in every 3 weeks, then a maintenance dose of $10 \mathrm{mg} / \mathrm{kg}$ every 12 weeks, until disease progression or inacceptable toxicity. Based on preclinical evidences that early release of tumor antigens and activation of tumor-specific $\mathrm{T}$ cells following SRS may enhance the effects of immunotherapy $[16,17]$, ipilimumab and nivolumab were generally administered 48-72 hours before receiving SRS. The choice of treatment was mainly based on the availability of checkpoint inhibitors for clinical standard practice in Italy. For patients with metastatic melanoma, the Italian Medicine Agency (AIFA) approved ipilimumab in February 2013 and nivolumab in March 2016. This means that ipilimumab was the only choice between 2013 and 2016, while nivolumab has been used more frequently since 2016 in patients with either BRAF wild-type melanoma or who had previously received BRAF/MEK inhibitors and ipilimumab. Salvage therapies at progression were chosen by the treating physicians; selected patients with clinical benefits from systemic treatments were allowed to continue nivolumab beyond progression.

Patients were clinically examined approximately at 2-6 weeks intervals. At each visit, neurological status and severity of complications were recorded according to the Common Terminology Criteria for Adverse Events 4.0. MRI was made every 2 months in the first year after the treatment, and subsequently every 2-3 months or as appropriate. For brain metastases measuring $\geq 5 \mathrm{~mm}$, intracranial complete response $(\mathrm{CR})$, partial response $(\mathrm{PR})$, stable disease $(\mathrm{SD})$, and progressive disease (PD) were determined by MRI according to the modified response evaluation criteria in solid tumors criteria (mRECIST v1.1.) [18], with tumor measurements and reporting of scans carried out by the same neuroradiologist (A.B.). Pseudoprogression was defined as transient increased contrast enhancement and edema occurring few months from SRS which resolved or stabilized during subsequent follow-up. Extracranial response was assessed according to RECIST v1.1. [19]. Diagnosis of tumor progression or $\mathrm{RN}$ were determined on the basis of histological findings (for patients who underwent surgical resection) or with imaging using MRI and 3,4-dihydroxy-6-(18) F-fluoro-l-phenylalanine-(F-DOPA)-PET-CT, as previously reported [20].

\section{Outcomes and data analysis}

Primary endpoint was intracranial PFS. Secondary endpoints were extracranial PFS, OS, and neurological toxicity. Time-to-event analysis were estimated using the Kaplan-Meier method from the date of SRS. Chi-square and non-parametric Mann-Whitney tests were used to examine between-group covariate differences, and the Cox proportional hazards model was employed for univariate and multivariate analysis to assess the effects of clinical/treatment variables on outcomes. Variables included in the univariate analysis were age at diagnosis, gender, KPS score, previous systemic treatments, number of metastases, extracranial disease status, diagnosisspecific graded prognostic assessment (DS-GPA) score [21], type of SRS, total tumor volume, GTV, and PTV. Variables at significance levels of $p<0.05$ were included in multivariate analysis. Standard softwares were used for statistical analysis (SAS software, version 9.3; XLSTAT).

\section{Results}

\section{Patient characteristics}

A total of 80 consecutive patients with 326 untreated melanoma brain metastases who received SRS for 1-10 lesions combined with ipilimumab or nivolumab were analyzed. Patient characteristics are shown in Table 1. Forty-five patients received concurrently SRS and ipilimumab, and 35 patients SRS and nivolumab with a median interval between infusion and SRS of 3 days (range 2-7 days). There were no significant differences between groups in terms of gender, age, number of metastases, KPS scores, irradiated volumes, DS-GPA, and type of SRS (single-fraction or multi-fraction SRS). Forty-one patients received multi-fraction SRS for at least one metastasis. Fifty-six patients with extracranial metastases had one or two lines of systemic therapy prior to SRS; among them, twenty-eight patients with BRAF-mutated tumors were previously treated with BRAF/MEK inhibitors, and 7 patients received ipilimumab.

For progressive disease, 27 patients received subsequent systemic therapy, including chemotherapy (SRS and ipilimumab, 8; SRS and nivolumab, 4), BRAF/MEK inhibitors (SRS and nivolumab, 3), and checkpoint inhibitors (SRS and ipilimumab, 8; SRS and nivolumab, 4). Eight patients who progressed on ipilimumab received nivolumab or pembrolizumab, whereas 7 patients in SRS and nivolumab group received trametinib and dabrafenib or combined ipilimumab and fotemustine as salvage therapies. In addition, 7 asymptomatic patients with good performance status continued nivolumab administration beyond intracranial progression. At the time of intracranial progression, local salvage therapies included surgery $(n=9)$, SRS $(n=29)$, and WBRT $(n=8)$. Dexamethasone up to $4 \mathrm{mg}$ per day for more than 2 weeks was given in 37 patients at the time of SRS $(n=23)$ or to manage toxicity $(n=14)$. At the time of analysis (July 2018), 17 patients were still undergoing treatment; 51 (64\%) had died.

\section{Progression-free survival and survival}

With a median follow-up of 15 months, 32 (71\%) out of 45 patients in SRS and ipilimumab group, and 20 (57\%) out of 35 patients in SRS and nivolumab group had an intracranial progression event, with a median 
Table 1 Patient characteristics and treatment parameters

\begin{tabular}{|c|c|c|c|}
\hline \multirow[t]{2}{*}{ Variable } & \multirow{2}{*}{$\begin{array}{l}\text { SRS and } \\
\text { ipilimumab } \\
N=45\end{array}$} & \multicolumn{2}{|l|}{$\begin{array}{l}\text { SRS and } \\
\text { nivolumab }\end{array}$} \\
\hline & & $N=35$ & $\mathrm{p}$ \\
\hline $\operatorname{Sex}(F / M)$ & $17 / 28$ & $14 / 21$ & 1.0 \\
\hline \multicolumn{4}{|l|}{ Age (years) } \\
\hline median & 54 & 56 & 0.2 \\
\hline range & $23-78$ & $26-80$ & \\
\hline KPS & & & 0.8 \\
\hline median & 80 & 80 & \\
\hline $60-70$ & 13 & 9 & \\
\hline $80-100$ & 32 & 26 & \\
\hline BRAF mutation & & & 0.8 \\
\hline present & 15 & 13 & \\
\hline absent & 30 & 22 & \\
\hline undetermined & & & \\
\hline Extracranial disease & & & 0.7 \\
\hline present & 34 & 25 & \\
\hline absent & 11 & 10 & \\
\hline Number of metastases & & & 0.4 \\
\hline single & 8 & 9 & \\
\hline multiple & 37 & 26 & \\
\hline DS-GPA & & & 0.3 \\
\hline $0-1$ & 9 & 6 & \\
\hline $1.5-2.5$ & 22 & 16 & \\
\hline $3-4$ & 14 & 13 & \\
\hline Type of SRS & & & 0.76 \\
\hline Single-fraction SRS & 153 & 132 & \\
\hline Fractionated SRS & 22 & 19 & \\
\hline Size of metastases & & & 0.8 \\
\hline$<2 \mathrm{~cm}$ & 99 & 84 & \\
\hline $2-3 \mathrm{~cm}$ & 46 & 38 & \\
\hline$\geq 3 \mathrm{~cm}$ & 30 & 29 & \\
\hline Total tumor volume $\left(\mathrm{cm}^{3}\right)$ & & & 0.1 \\
\hline median & 7.4 & 9.2 & \\
\hline range & $0.5-33.1$ & $0.7-33$ & \\
\hline $\operatorname{GTV}\left(\mathrm{cm}^{3}\right)$ & & & 0.6 \\
\hline median & 1.12 & 1.2 & \\
\hline range & $0.05-27.9$ & $0.4-31.2$ & \\
\hline PTV $\left(\mathrm{cm}^{3}\right)$ & & & 0.3 \\
\hline median & 1.71 & 1.83 & \\
\hline range & $0.1-39.1$ & $0.09-42.6$ & \\
\hline Conformity index ${ }^{a}$ & & & 0.5 \\
\hline median & 1.43 & 1.41 & \\
\hline range & $1.10-1.91$ & $1.12-1.85$ & \\
\hline
\end{tabular}

SRS stereotactic radiosurgery, KPS Karnofsky Performance Status DS-GPA Diagnosis-Specific Graded Prognostic Factors, GTV Gross Target Volume

PTV Planning Target Volume, ${ }^{\text {a }}$ calculated as prescribed isodose volume/tumor volume

encompassed by the prescription isodose volume intracranial PFS of 6 and 10 months $(p=0.02)$, respectively. The 6-month and 12-month intracranial PFS rates were $69 \%(95 \% \mathrm{CI}, 54-87 \%)$ and $42 \%(95 \% \mathrm{CI}$, 24-65\%) in SRS and nivolumab group and 48\% (95\%CI, 34-64\%) and 17\% (95\%CI, 5-31\%) in SRS and ipilimumab group ( $p=0.02)$, respectively, (Fig. 1a). Median OS was 22.0 months in SRS and nivolumab group and 14.7 months in SRS and ipilimumab group $(p=0.015)$ (Fig. $1 \mathrm{~b})$; respective 12-month and 24-month survival probabilities were $78 \%(95 \% \mathrm{CI}, 63-95 \%)$ and $42 \%(95 \% \mathrm{CI}$, $26-63 \%)$, and $68 \%(95 \% \mathrm{CI}, 51-89 \%)$ and $20 \%(95 \% \mathrm{CI}$, 5-36\%). Twenty-three patients succumbed to their intracranial disease (SRS and ipilimumab, 15; SRS and nivolumab, 8) and 28 patients died of progressive extracranial disease (SRS and ipilimumab, 17; SRS and nivolumab, 11).

Analysis of LC and distant brain control (DBC) showed significant differences by groups (Fig. 2). Four patients who received SRS and nivolumab and 10 who were treated with SRS and ipilimumab had local failure; 6-month and 12-month LC rates were 96\% (95\%CI, $87-100 \%)$ and $85 \%$ (95\%CI, 75-95\%) in SRS and nivolumab group, respectively, and $90 \%(95 \% \mathrm{CI}, 81-99 \%)$ and $70 \%$ (95\%CI, 59-81\%) in SRS and ipilimumab group, respectively $(p=0.03)$. With a median time of 4 months, CR and PR occurred in $41 \%$ and $35 \%$ of patients receiving SRS and nivolumab, and $23 \%$ and $37 \%$ in those receiving SRS and ipilimumab, yielding to intracranial objective response rates of $76 \%$ and $60 \%$. LC was similar for symptomatic and asymptomatic lesions. DBC rates were significantly different; $75 \%(95 \% \mathrm{CI}, 59-93 \%)$ and $46 \%$ (95\% CI, 29-65\%) in SRS and nivolumab group and $52 \%(95 \% \mathrm{CI}, 34-69 \%)$ and $20 \%(95 \% \mathrm{CI}, 6-35 \%)$ in SRS and ipilimumab group at 6 and 12 months, respectively $(p=0.027)$.

The 6-month and 12-month extracranial PFS rates were $57 \%$ and $37 \%$ in SRS and nivolumab group and $42 \%$ and $17 \%$ in SRS and ipilimumab group $(p=0.03)$, respectively; global PFS rates were 53\% and $36 \%$ and $34 \%$ and $17 \%(p=0.02)$, (Additional file 1: Figure S1). The majority of patients had concurrent intracranial and extracranial progression; intracranial progression alone occurred in 5 patients receiving SRS and ipilimumab and 2 patients undergoing SRS and nivolumab.

\section{Analysis of prognostic factors}

For the whole population, multivariate analysis showed that SRS and nivolumab treatment, multi-fraction SRS, absent extracranial disease, and KPS $>70$ were significant indices of prolonged OS (Table 2). According to DS-GPA score, median OS was 6.8, 14.2 and 29.0 months for patients with scores of 0-1, 1-2.5, and 3-4 ( $p$ $<0.001)$, respectively. Patients who had received BRAF 

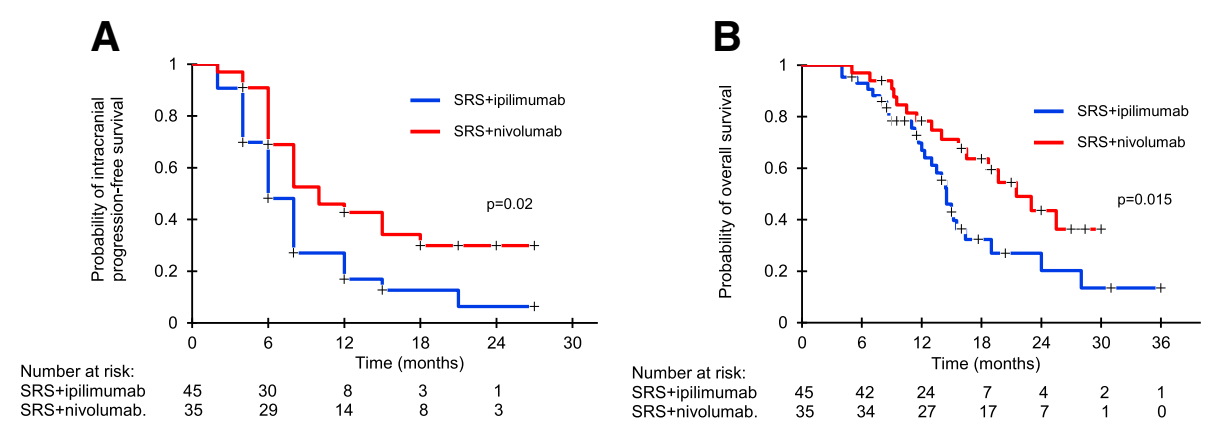

Fig. 1 Kaplan-Meier analysis of overall survival (OS, a) and intracranial progression-free survival (PFS, b) for patients receiving concurrent SRS and ipilimumab (blue line) or nivolumab (red line). OS and intracranial PFS were significantly better in SRS and nivolumab group

and MEK inhibitors treatment prior to the study showed a trend toward worse survival $(p=0.07)$ : for this group, 12-month and 24-month OS rates were $46 \%$ and 16\%, respectively. Multi-fraction SRS was associated with better intracranial PFS; 6-month and 12-month rates were $70 \%$ and $40 \%$ for patients receiving multi-fraction SRS and $46 \%$ and $10 \%$ for those undergoing single-fraction SRS $(p=0.01)$, respectively (Fig. 3 ). Groups sub-analysis showed that patients receiving multi-fraction SRS and nivolumab had better intracranial PFS (Fig. 3b). The use of steroids showed a trend toward worse OS (HR 1.74, 95\%CI, 0.94-2.2; $p=0.057)$ and intracranial PFS (HR1.97, 95\%CI, 0.91-2.4; $p=0.068$ ) (Additional file 2: Figure S2). Aside from combined SRS and nivolumab, no other factors, including tumor size, irradiated volumes, number of lesions, and SRS type were predictor of LC. Concurrent nivolumab and SRS type resulted in better extracranial PFS rates; 6-month and 12-month rates were $61 \%$ and $40 \%$ in patients receiving multi-fraction SRS and $47 \%$ and $18 \%$ in those receiving single-fraction SRS, respectively $(p=0.03)$.

\section{Toxicity}

A clinical neurological improvement after SRS occurred in $15(63 \%)$ out of 22 patients with pre-existing neurological symptoms. Adverse events were recorded in
$66.6 \%$ of patients having SRS and ipilimumab and 57\% of those receiving SRS and nivolumab, with grade 3 events observed in $11(24 \%)$ and $6(17 \%)$ patients, respectively (Table 3 ). CNS-related grade 3 events were represented by headache $(\boldsymbol{n}=3)$, seizure $(\boldsymbol{n}=3)$, and brain edema $(\boldsymbol{n}=4)$. Intracranial hemorrhage was seen in 5 patients, being symptomatic in two (grade 3). Ipilimumab was discontinued in 5 patients and nivolumab in 3 patients. The most common extracranial grade 3 events were diarrhoea $(\boldsymbol{n}=4)$, increased serum aspartate aminotransferase $(\boldsymbol{n}=3)$, and rash [2].

The risk of radiation-induced brain necrosis (RN) was evaluated by MRI/F-DOPA PET-CT studies. With a median time of 8 weeks (range 4-16 weeks), an early enlargement of irradiated lesions was recorded in 19 patients (43 lesions). Grade 3 neurological deficits related to imaging changes developed in 6 patients requiring medical therapy (Table 3 ). On subsequent imaging, tumor volumes decreased $(\boldsymbol{n}=19)$ or disappeared $(\boldsymbol{n}=21)$ in 13 patients at a median time of 8 weeks, confirming the diagnosis of pseudoprogression, and continued to enlarge in 3 patients who were recorded as having intracranial progression. Imaging criteria suggestive of RN were observed in further $12 / 80$ patients (35/ 326 lesions) who were followed up for at least 6 months with MRI and DOPA PET-CT scans; SRS and nivolumab,
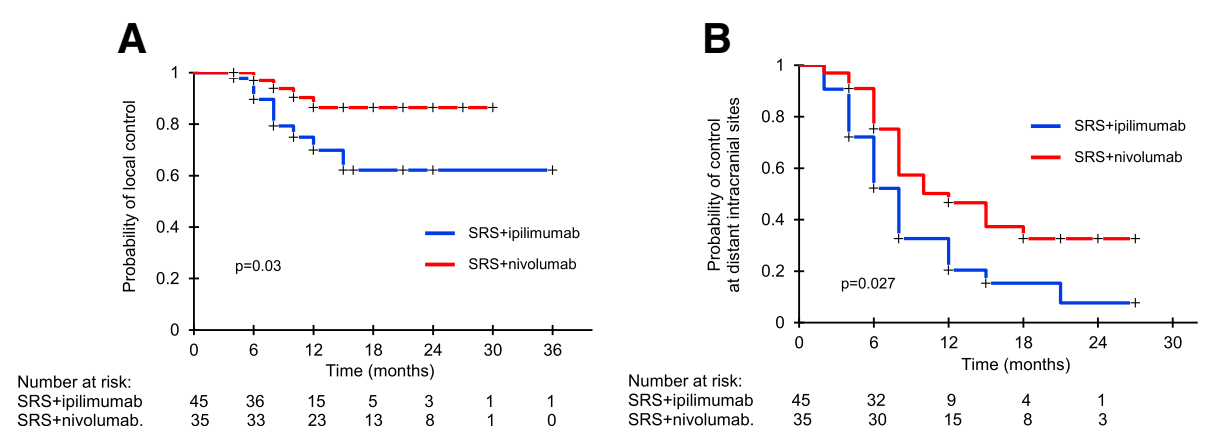

Fig. 2 Kaplan-Meier analysis of local control (LC, a) and distant brain control (DBC, b) after concurrent SRS and ipilimumab (blue line) or nivolumab (red line). LC and DBC were significantly better in SRS and nivolumab group 
Table 2 Independent favorable prognostic factor for intracranial PFS* and OS

\begin{tabular}{lllll}
\hline Outcome & Variable & Hazard ratio & $95 \% \mathrm{Cl}$ & $\mathrm{P}$ \\
\hline Intracranial PFS & SRS and nivolumab & 0.54 & $0.32-0.92$ & $0.28-0.87$ \\
& fmulti-fraction SRS & 0.48 & 0.038 \\
OS & SRS and nivolumab & 0.51 & $0.28-0.81$ & 0.015 \\
& multi-fraction SRS & 0.54 & $0.33-0.96$ & 0.043 \\
& KPS $>70$ & 0.34 & $0.23-0.78$ & 0.010 \\
& absent extracranial disease & 0.50 & $0.29-0.81$ & 0.018
\end{tabular}

Abbreviations: OS, overall survival; PFS, progression-free survival; $\mathrm{HR}$, hazard ratio; $\mathrm{Cl}$, confidence interval; *Variables with a significance of $p<0.05$ at univariate analysis were included in the multivariate analysis. The following variables were evaluated: age, gender, Karnofsly Perforance Status (KPS) score, histology, extracranial disease status, systemic therapy, number of metastases, time to brain metasases development, conformity index, and irradiated volumes

5/35; SRS and ipilimumab, 7/45). In 5 patients who underwent surgery, diagnosis of RN was confirmed by histology. The estimated 12-month incidence of RN was 25\% in SRS and ipilimumab group and $17 \%$ in SRS and nivolumab group ( $p=0.15$ ); RN was symptomatic in 12 patients (SRS and ipilimumab, $n=7$; SRS and nivolumab, $n=5$ ), requiring surgery or long-term medical treatment. Grade 2 or 3 neurotoxicity, including motor deficits $(n=7)$, neurocognitive deficits $(n=3)$, seizure $(n=2)$, and speech deficits $(n=1)$, occurred in 7 and 2 patients, respectively.

No factors were independent predictors of RN, including tumor size, total tumor volume, GTV, PTV, and type of SRS; however, the median GTV was higher for symptomatic necrotic lesions (7.3 vs $2.7 \mathrm{ml} ; p=0.003)$. The risk was similar after single-fraction SRS or multi-fraction SRS, even though the median GTV was significantly higher for lesions treated with multi-fraction SRS (11.7 vs $1.8 \mathrm{ml} ; p=0.0001)$; for lesions $>2.0 \mathrm{~cm}$ in size, the 12-month estimated risk of RN was $28 \%$ and $16 \%$ after single-fraction SRS and multi-fraction SRS $(p=0.07)$, respectively.

\section{Discussion}

Results of this study show that SRS concurrently to nivolumab or ipilimumab has a meaningful intracranial efficacy in patients with either asymptomatic or symptomatic untreated melanoma brain metastases. The 6-month and 12-month intracranial PFS rates were $69 \%$ and $42 \%$ for patients undergoing SRS and nivolumab and $48 \%$ and $17 \%$ for those receiving SRS and ipilimumab $(p=0.02)$, respectively. Combined SRS and nivolumab was associated with significantly longer LC and DBC; local failure rates decreased from $10 \%$ to $4 \%$ and from $30 \%$ to $14 \%$, and DBF rates from $48 \%$ to $25 \%$ and from $80 \%$ to $54 \%$ at 6 months and 12 months, respectively. Similarly, extracranial PFS and OS were significantly better with SRS and nivolumab, with more than $40 \%$ of patients expected to be alive at 2 years.

Our findings are consistent with results from recent retrospective series on the efficacy of combining SRS with checkpoint inhibitors (Table 4) [22-32]. In a series of 96 patients with 314 melanoma brain metastases who had SRS within 3 months from receiving different systemic therapies, Ahmed et al. [24] observed 12-month DBC rates of $38 \%$ and $21 \%$ after SRS and nivolumab or ipilimumab, respectively, and improved survival compared to conventional chemotherapy. In another series of 46 patients with a total of 113 melanoma brain metastases, Kiess et al. [23] reported an estimated 12-month LC, DBC and OS rates of about $87 \%, 37 \%$ and $60 \%$, respectively, in patients receiving SRS before or
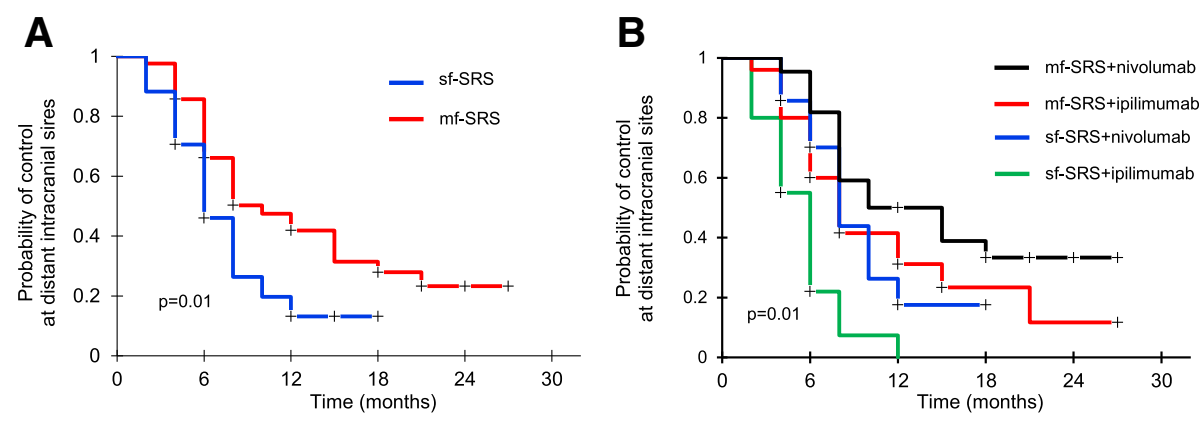

Fig. 3 Kaplan-Meier analysis of intracranial progression-free survival (PFS) after single-fraction radiosurgery (sf-SRS, blue line) or multi-fraction SRS (mf-SRS, red line) in combination with ipilimumab or nivolumab. Patients receiving mf-SRS had significantly better intracranial PFS than those treated with sf-SRS (a); differences in PFS were seen in both ipilimumab and nivolumab groups (b) 
Table 3 Adverse events

\begin{tabular}{|c|c|c|c|c|}
\hline & \multicolumn{2}{|c|}{ SRS and Ipilimumab $(n=45)$} & \multicolumn{2}{|c|}{ SRS and Nivolumab $(n=35)$} \\
\hline & Grade 1 or 2 & Grade 3 & Grade 1 or 2 & Grade 3 \\
\hline Number of patients with at least an adverse event ${ }^{a}$ & $31(68 \%)$ & $11(24 \%)$ & $20(57 \%)$ & $6(17 \%)$ \\
\hline \multicolumn{5}{|l|}{ Event } \\
\hline Diarrhoea & $11(24 \%)$ & $3(7 \%)$ & $5(14 \%)$ & $1(3 \%)$ \\
\hline Nausea or vomiting & $8(18 \%)$ & $1(2 \%)$ & $4(12 \%)$ & $1(3 \%)$ \\
\hline Constipation & $5(11 \%)$ & 0 & $2(6 \%)$ & 0 \\
\hline Increased AST and/or ALT levels & $4(9 \%)$ & $2(4 \%)$ & $4(12 \%)$ & $2(6 \%)$ \\
\hline Fatigue & $12(27 \%)$ & $3(7 \%)$ & $6(18 \%)$ & $2(6 \%)$ \\
\hline Endocrine immune disorders & $3(7 \%)$ & 0 & $2(6 \%)$ & 0 \\
\hline Rash/Pruritus & $10(22 \%)$ & $1(2 \%)$ & $6(18 \%)$ & $1(3 \%)$ \\
\hline Arthralgia & $5(11 \%)$ & 0 & $3(9 \%)$ & 0 \\
\hline Muscle weakness right or left sided & $3(7 \%)$ & $1(2 \%)$ & $2(6 \%)$ & $1(3 \%)$ \\
\hline \multicolumn{5}{|l|}{ CNS event } \\
\hline Headache & $8(18 \%)$ & $2(4 \%)$ & $4(12 \%)$ & $1(3 \%)$ \\
\hline Hemorrhage & $3(7 \%)$ & $1(2 \%)$ & $2(6 \%)$ & $1(3 \%)$ \\
\hline Seizure & $3(7 \%)$ & $2(4 \%)$ & $2(6 \%)$ & $1(3 \%)$ \\
\hline Diziness & $4(9 \%)$ & 0 & $2(6 \%)$ & 0 \\
\hline Brain necrosis & $13(29 \%)$ & $5(11 \%)$ & $7(20 \%)$ & $3(9 \%)$ \\
\hline Discontinuation of treatment & 5 & & 3 & \\
\hline
\end{tabular}

${ }^{a}$ Treatment-related adverse events of any grade occurring in at least $5 \%$ of patiens in either cohorts. Some patients had more than one event. No grade 4 events were reported in both cohorts

concurrently to ipilimumab. A similar efficacy of SRS and pembrolizumab has been reported in other few studies [25, 31-33]. With regard to the treatment sequencing, published results suggest that SRS and checkpoint inhibitors given concurrently, typically within 4 weeks of SRS, are associated with improved intracranial control and survival compared to nonconcurrent therapy $[23,25,29,31,34]$ or SRS given alone [22, 26], with no significantly increased neurotoxicity. Overall, our results provide further evidence supporting the efficacy of concurrent immunotherapy and SRS for melanoma brain metastases, even in patients with symptomatic and large lesions.

Several factors had a positive impact on patient outcomes. An intriguing finding of our study was the significantly better intracranial and extracranial PFS at 6 and 12 months in patients receiving multi-fraction SRS and concurrent checkpoint inhibitors, either nivolumab or ipilimumab. Emerging evidence suggests that radiotherapy and immunotherapy may have synergistic effects [35-44]. Preclinical studies have shown that the combination of radiotherapy and targeted PD-1/PD-L1 therapy activates cytotoxic T-cells, reduces myeloid-derived suppressor cells, and may induce an abscopal response, as defined by a significant growth inhibition of the tumor outside the irradiated field [35-37]. Similarly, several preclinical and clinical studies have reported the enhanced immunostimulatory effects of radiotherapy when given in combination with anti-CTL-4 antibodies for either irradiated or non-irradiated tumors [38-44]. Dewan et al. [39] demonstrated that an abscopal effect occurred only in mice treated with anti-CTLA-4 antibodies combined to multi-fraction SRS ( $3 \times 8$ Gy), but not to single-fraction SRS (20 Gy). Consistent with this finding, abscopal responses have been reported in patients receiving hypofractionated radiotherapy and ipilimumab [40-44].

Although our results support the synergistic effects between multi-fraction SRS and either ipilimumab or nivolumab, large prospective studies are required to confirm our findings. Currently, there are no prospective controlled data showing that adding radiotherapy, either SRS or fractionated radiotherapy, to PD-1/PD-L1 or CTLA-4 inhibition may enhance abscopal responses. The question whether the combination of checkpoint inhibition and radiotherapy improves the efficacy of checkpoint inhibition alone in different tumors is being addressed in ongoing clinical trials [45].

The management paradigm of melanoma brain metastases is rapidly changing. Both PD-1/PD-L1 or CTLA-4 checkpoint inhibitors have shown activity in patients with melanoma brain metastases, with a response rate of up to one third of patients [9-11]. More recently, a 
Table 4 Selected studies assessing the efficacy and toxicity of SRS and immunotherapy for the treatment of melanoma brain metastases

\begin{tabular}{|c|c|c|c|c|c|c|}
\hline Authors & Patients (n) & Treatment & $\begin{array}{l}\text { Median survival } \\
\text { (months) }\end{array}$ & Brain control & Neurotoxicity & $\begin{array}{l}\text { Brain necrosis } \\
\text { (\% of patients) }\end{array}$ \\
\hline \multirow{3}{*}{$\begin{array}{l}\text { Knisely et al., } \\
2012 \text { [22] }\end{array}$} & 16 & Ipi after SRS & 21.3 & NR & NR & NR \\
\hline & 11 & Ipi before SRS & 19.8 & NR & NR & NR \\
\hline & 50 & SRS alone & 4.9 & NR & NR & NR \\
\hline \multirow[t]{3}{*}{$\begin{array}{l}\text { Kiess et al., } \\
2015 \text { [23] }\end{array}$} & 15 & $\begin{array}{l}\text { Concurrent SRS and } \\
\text { Ipi (within } 1 \text { month) }\end{array}$ & 1 -year 65\% & 1-year LC 100\% & $\begin{array}{l}\text { Grade 2, 33\% } \\
\text { Grade 3, 26\% }\end{array}$ & \multirow{3}{*}{$\begin{array}{l}\text { Early and late RN } \\
50 \% \text { of patients } \\
\text { treated during or } \\
\text { before Ipi and 13\% } \\
\text { of patients treated } \\
\text { after Ipi. }\end{array}$} \\
\hline & 19 & $\begin{array}{l}\text { Nonconcurrent, SRS } \\
\text { before Ipi (median } \\
3 \text { months) }\end{array}$ & 1 -year 56\% & 1-year LC 87\% & $\begin{array}{l}\text { Grade 2, 10\% } \\
\text { Grade 3, 6\% } \\
\text { Grade 4, 3\% }\end{array}$ & \\
\hline & 12 & $\begin{array}{l}\text { Nonconcurrent, SRS } \\
\text { after Ipi (median } 2 \\
\text { months) }\end{array}$ & 1 -year 40\% & 1-year LC 89\% & & \\
\hline $\begin{array}{l}\text { Ahmed et al., } \\
2016 \text { [24] }\end{array}$ & 26 & SRS/SRT and Nivo & $78 \%$ (1-year 55\%) & $\begin{array}{l}\text { 6-month and } 1 \text {-year } \\
\text { DBC } 61 \text { and } 38 \% \\
6 \text {-month and } 1 \text {-year } \\
\text { LC } 89 \text { and } 82 \%\end{array}$ & Grade $2,37 \%$ & $27 \%$ \\
\hline \multirow[t]{2}{*}{$\begin{array}{l}\text { Qian et al., } \\
2016 \text { [25] }\end{array}$} & 33 & $\begin{array}{l}\text { SRS and concurrent } \\
\text { IPI (1) or Pembro (14) }\end{array}$ & 19.1 & NR & NR & NR \\
\hline & 42 & $\begin{array}{l}\text { Nonconcurrent SRS } \\
\text { and Ipi (35) or } \\
\text { Pembro (7) }\end{array}$ & 9 & NR & NR & NR \\
\hline \multirow[t]{2}{*}{$\begin{array}{l}\text { Choong et al., } \\
2017 \text { [26] }\end{array}$} & 28 & $\begin{array}{l}\text { Concurrent SRS and } \\
\text { Ipi (within } 6 \text { weeks) }\end{array}$ & 7.5 (1-year 40\%) & 7.5 months & NR & $0 \%$ \\
\hline & 11 & $\begin{array}{l}\text { Concurrent SRS and } \\
\text { Nivo (within } 6 \text { weeks) }\end{array}$ & 20.4 (1-year 78\%) & 12.7 months & NR & $18 \%$ \\
\hline \multirow[t]{2}{*}{$\begin{array}{l}\text { Cohen-Inbar } \\
\text { et al., } 2017 \text { [27] }\end{array}$} & 32 & $\begin{array}{l}\text { Ipi before or during } \\
\text { SRS }\end{array}$ & 1-year 59.4\% & $\begin{array}{l}\text { 1-year LC and DBF } \\
54.4 \text { and } 15.8 \%\end{array}$ & NR & $31 \%$ \\
\hline & 14 & Ipi after SRS & 1 -year 33\% & $\begin{array}{l}\text { 1-year LC and DBF } \\
16.5 \text { and } 26.8 \%\end{array}$ & NR & $7 \%$ \\
\hline $\begin{array}{l}\text { Gaudi-Marqueste } \\
\text { et al., } 2017 \text { [28] }\end{array}$ & 21 & $\begin{array}{l}\text { SRS before Ipi (21), } \\
\text { Nivo (17), both (6) }\end{array}$ & $\begin{array}{l}\text { Ipi, } 8.6 \text { (1-year } \\
41.2 \%) \text { Nivo,12 } \\
(1-\text { year 63\%) }\end{array}$ & NR & NR & NR \\
\hline $\begin{array}{l}\text { Patel et al., } \\
2017 \text { [29] }\end{array}$ & 20 & $\begin{array}{l}\text { Ipi plus SRS (whitin } \\
4 \text { months) }\end{array}$ & 8 (1-year 37.1\%) & $\begin{array}{l}1 \text {-year LC and DBF } \\
71 \text { and } 12 \%\end{array}$ & NR & $18 \%$ at 1 year \\
\hline $\begin{array}{l}\text { Skrepnik et al., } \\
2017 \text { [30] }\end{array}$ & 25 & $\begin{array}{l}\text { Ipi before or } \\
\text { concurrent (within } \\
1 \text { month) }\end{array}$ & $\begin{array}{l}35(1-y e a r \text { and } 2- \\
\text { year } 83 \text { and } 64 \%)\end{array}$ & $\begin{array}{l}16.7(1-y e a r \text { and } \\
2 \text {-year } 52 \text { and 34.8\%) }\end{array}$ & NR & $20.75 \%$ symptomatic \\
\hline \multirow[t]{2}{*}{$\begin{array}{l}\text { Chen et al., } \\
2018 \text { [31] }\end{array}$} & $23(28)^{\circ}$ & $\begin{array}{l}\text { concurrent SRS-SRT } \\
\text { and Ipi or Pembro }\end{array}$ & 24.7 (1-year 75\%) & 1-year LC 88\% & $\begin{array}{l}\text { Grade 2, 42\% } \\
\text { Grade 3,0\% }\end{array}$ & $\begin{array}{l}27 \% \text { of } 22 \text { metastases } \\
\text { confirmed by histology }\end{array}$ \\
\hline & $12(51)^{\circ}$ & $\begin{array}{l}\text { Nonconcurrent } \\
\text { SRS-SRT and Ipi } \\
\text { or Pembro }\end{array}$ & 14.5 (1-year 53\%) & 1-year LC 79\% & $\begin{array}{l}\text { Grade 2, 35\% } \\
\text { Grade 3, 33\% }\end{array}$ & \\
\hline $\begin{array}{l}\text { Nardin et al., } \\
2018 \text { [32] }\end{array}$ & 25 & SRS and Pembro & 15.3 & 8.4 (6-months LC 80\%) & NR & $6.8 \%$ \\
\hline \multirow[t]{2}{*}{ Current series } & 45 & $\begin{array}{l}\text { concurrent SRS-SRT } \\
\text { and Nivo (within } 1 \\
\text { week) }\end{array}$ & 22 (1-year 78\%) & $\begin{array}{l}\text { 1-year } 42 \% \text { 1-year LC } \\
\text { and DBC } 85 \text { and } 46 \%\end{array}$ & Grade $3,11 \%$ & $25 \%$ at 1-year \\
\hline & 35 & $\begin{array}{l}\text { Concurrent SRS-SRT } \\
\text { and Ipi (within } 1 \\
\text { week) }\end{array}$ & 14.7 (1-year 68\%) & $\begin{array}{l}\text { 1-year 17\% 1-year LC } \\
\text { and DBC } 70 \text { and 20\% }\end{array}$ & Grade 3, 6\% & $17 \%$ at 1 year \\
\hline
\end{tabular}

Ipi Ipilimumab, Nivo Nivolumab, Pembro Pembrolizumab, SRS Stereotactic radiosurgery, SRT Stereotactic radiotherapy, LC local control, DBC distant brain control, $N R$ not reported; ${ }^{\circ}$ Study including patients with brain metastases from melanoma, non small-cell lung cancer, and renal cell carcinoma 
combination of checkpoint inhibitors has been explored as a new strategy to improve the outcome over monotherapy. Two prospective trials assessing the efficacy and safety of combining nivolumab and ipilimumab in patients with asymptomatic melanoma brain metastases have showed durable intracranial response in about $65 \%$ of patients [11, 12]; however, grade 3 or 4 occurred in more than $50 \%$ of patients causing interruption of treatment in up to $26 \%$ of patients. Even though toxicity of combined checkpoint inhibitors occurs in a significant proportion of patients, systemic therapy alone may represent a reasonable initial approach for asymptomatic brain metastases; however, its efficacy in symptomatic lesions remains to be proven. In absence of controlled randomized trials, results observed in our study suggest that combined immunotherapy and SRS should be considered in the setting of large symptomatic melanoma brain metastases. Notably, efficacy of treatments was apparently maintained in patients receiving corticosteroids, for whom the response to immunotherapy alone seems to be less effective $[9,46]$.

Treatments were generally well tolerated. Early or late radiological changes suggestive of $\mathrm{RN}$ were shown in one third of patients, with grade 3 neurotoxicity occurring in $9 \%$ of them. The risk was consistent with those observed in other series of concurrent checkpoint inhibitors and SRS [24, 26, 27, 29, 30]. Notably, most of radiological changes occurred in the first 3-4 months after SRS and were typically characterized by an enlargement of enhanced lesions and increased perilesional edema, so called pseudoprogression. Radiological findings resolved in 6-8 weeks in the majority of patients and were rarely associated with neurological symptoms; however, a strict follow-up imaging is recommended in these patients for distinguishing pseudoprogression from true tumor progression. Even though the risk of RN after concurrent therapy is similar to that observed with SRS alone [14, 47, 48], the absence of RN after combined ipilimumab and nivolumab [11, 12] addresses important questions about the optimal treatment strategy for patients with melanoma brain metastases. The use of SRS as up-front or salvage therapy to maximize benefit and minimize toxicity needs to be explored in future trials.

The current study has several limitations, owing to its retrospective nature. The presence of unmeasured baseline characteristics, such as presence of comorbidities, levels of PD-1/PD-L1 expression, extension of extracranial disease, and previous systemic treatments is likely to introduce selection bias. Moreover, different doses and duration of corticosteroids for controlling neurological symptoms, or different salvage therapies at progression may contribute to the observed differences in clinical outcomes between groups. Nevertheless, our results demonstrate that concurrent SRS and nivolumab or ipilimumab is associated with high intracranial activity.
In conclusion, our study shows that SRS combined with nivolumab provides better intracranial control than SRS and ipilimumab in patients with both symptomatic and asymptomatic melanoma brain metastases, although a significant subset of patients receiving immunotherapy and concurrent SRS may develop symptomatic RN. Combination of nivolumab with multi-fraction SRS has the potential to provide a strong synergistic effect. The efficacy and safety of different radiation schedules and checkpoint inhibitors over other therapeutic strategies require further investigation.

\section{Additional files}

Additional file1: Figure S1. Kaplan-Meier analysis of extracranial progression-free survival $(\mathrm{S} 1, \mathrm{~A})$ and global progression-free survival $(\mathrm{S1}, \mathrm{B})$ for patients receiving SRS and ipilimumab (blue line) or nivolumab (red line). 6-month and 1-year extracranial PFS rates were 57 and 37\% and 42 and $17 \%$, respectively, in SRS and ipilimumab or nivolumab group. Respective 6-month and 1-year global PFS rates were 53 and $36 \%$ and 34 and $17 \%$. (PDF $255 \mathrm{~kb}$ )

Additional file 2: Figure S2. Kaplan-Meier analysis of intracranial progression-free survival (PFS) and overall survival (OS) in patients who received dexamethasone (yes, blue line) or not (not, red line) during treatments. For PFS, 6-month and 12-month rates were 42.3 and 24.2\%, and 73.6 and $35.9 \%$ respectively, in patients receiving dexamethasone or not. For OS, respective rates were 91.2 and $57.3 \%$ and 96 and $76.1 \%$. (PDF $40 \mathrm{~kb}$ )

\section{Abbreviations}

BRAF: Serine/threonine-protein kinase B-Raf; CNS: Central nervous system; CR: Complete response; CT: Computed tomography; CTLA-4: Cytotoxic Tlymphocyte antigen-4; CTV: Clinical target volume; DBC: Distant brain control: DS-GPA: Diagnosis-Specific graded prognostic assessment; GTV: Gross tumor volume; LC: Local control; MEK: Mitogen-activated protein kinase kinase; MRI: Magnetic resonance imaging; OS: Overall survival; PD: Progressive disease; PD-1: Programmed cell death 1; PD-L1: Programmed cell deathligands 1; PET: Positron emission tomography; PFS: Progression-free survival; PR: Partial response; PTV: Planning target volume; RN: Radiation-induced Brain Necrosis; SD: Stable disease; SRS: Stereotactic radiosurgery;

WBRT: Whole brain radiation therapy

\section{Acknowledgements}

The authors would like thank the patients presented in this study.

Funding

No funding sources are declared.

Availability of data and materials

Summarized datasets analyzed during the current study available from the corresponding author on reasonable request.

\section{Authors' contributions}

GM is responsible for the conception and design of the study, participated in the analysis and interpretation of data as responsible for statistical analysis, drafted and finally revised the article; DA has participated in the study and data analysis; CR has participated to the study and analysis of data; GCC has participated in the study and article preparation; LM has participated in the study; FB has participated in the study and article preparation; AB has participated in the study, analysis of data (imaging), and article preparation; $\mathrm{MO}$ has participated in the analysis, interpretation of data, and article preparation and revision; VE has participated in the study, article preparation and revision; PCG has participated in article preparation and revision. All authors read and approved the final manuscript. 


\section{Ethics approval and consent to participate}

The study approved by the Università di Roma Sapienza and Ospedale San Pietro Institutional Review Boards as retrospective clinical research. Due to the retrospective nature of the study and use of de-identified data, no consent was needed for the patients described in this series.

\section{Consent for publication}

Not applicable

\section{Competing interests}

GM has received personal fees from BrainLab, outside the submitted work. The remaining authors declare that they have no competing interests.

\section{Publisher's Note}

Springer Nature remains neutral with regard to jurisdictional claims in published maps and institutional affiliations.

\section{Author details}

'Radiation Oncology Unit, UPMC Hillman Cancer Center|, San Pietro Hospital FBF, 00189 Rome, Italy. ${ }^{2}$ Radiation Oncology Unit, Sant' Andrea Hospital, University Sapienza, 00100 Rome, Italy. ${ }^{3}$ IV Oncology Division, Istituto Dermopatico dell'Immacolata IRCCS, Rome, Italy. ${ }^{4}$ Neuroradiology Unit, Sant' Andrea Hospital, University Sapienza, 00189 Rome, Italy. ${ }^{5}$ RCCS Neuromed, 86077 Pozzilli (IS), Italy.

Received: 22 November 2018 Accepted: 2 April 2019

Published online: 11 April 2019

\section{References}

1. Ajithkumar T, Parkinson C, Fife K, et al. Evolving treatment options for melanoma brain metastases. Lancet Oncol. 2015;16:e486-97.

2. Kocher M, Soffietti R, Abacioglu U, et al. Adjuvant whole-brain radiotherapy versus observation after radiosurgery or surgical resection of one to three cerebral metastases: results of the EORTC 22952-26001 study. J Clin Oncol. 2011:29:134-41.

3. Brown PD, Jaeckle K, Ballman KV, et al. Effect of Radiosurgery Alone vs Radiosurgery With Whole Brain Radiation Therapy on Cognitive Function in Patients With 1 to 3 Brain Metastases: A Randomized Clinical Trial. JAMA. 2016:316:401-9.

4. Yu C, Chen JC, Apuzzo ML, et al. Metastatic melanoma to the brain: prognostic factors after gamma knife radiosurgery. Int J Radiat Oncol Biol Phys. 2002:52:1277-87.

5. Selek U, Chang EL, Hassenbusch SJ 3rd, et al. Stereotactic radiosurgical treatment in 103 patients for 153 cerebral melanoma metastases. Int J Radiat Oncol Biol Phys. 2004;59:1097-106.

6. Manon R, O'Neill A, Knisely J, et al. Phase II trial of radiosurgery for one to three newly diagnosed brain metastases from renal cell carcinoma, melanoma, and sarcoma: an Eastern Cooperative Oncology Group study ( $E$ 6397). J Clin Oncol. 2005;23:8870-6.

7. Minniti G, Paolini S, D'Andrea G, et al. Outcomes of postoperative stereotactic radiosurgery to the resection cavity versus stereotactic radiosurgery alone for melanoma brain metastases. J Neuro-Oncol. 2017; 132:455-62.

8. Davies MA, Saiag P, Robert C, et al. Dabrafenib plus trametinib in patients with BRAF(V600)-mutant melanoma brain metastases (COMBI-MB): a multicentre, multicohort, open-label, phase 2 trial. Lancet Oncol. 2017;18: 863-73.

9. Margolin K, Ernstoff MS, Hamid O, et al. Ipilimumab in patients with melanoma and brain metastases: an open-label, phase 2 trial. Lancet Oncol. 2012;13:459-65.

10. Goldberg SB, Gettinger SN, Mahajan A, et al. Pembrolizumab for patients with melanoma or non-small-cell lung cancer and untreated brain metastases: early analysis of a non-randomised, open-label, phase 2 trial. Lancet Oncol. 2016;17:976-83.

11. Long GV, Atkinson V, Lo S, Sandhu S, et al. Combination nivolumab and ipilimumab or nivolumab alone in melanoma brain metastases: a multicenter randomised phase 2 study. Lancet Oncol. 2018;19:672-81.

12. Tawbi HA, Forsyth PA, Algazi A, et al. Combined Nivolumab and Ipilimumab in Melanoma Metastatic to the Brain. N Engl J Med. 2018;379:722-30.
13. Lehrer EJ, Peterson J, Brown PD, et al. Treatment of brain metastases with stereotactic radiosurgery and immune checkpoint inhibitors: An international metanalysis of individual patient data. Radiother Oncol. 2018.

14. Minniti G, Scaringi C, Paolini S, et al. Single-Fraction Versus Multifraction ( $3 \times$ 9 Gy) Stereotactic Radiosurgery for Large ( $>2 \mathrm{~cm}$ ) Brain Metastases: A Comparative Analysis of Local Control and Risk of Radiation-Induced Brain Necrosis. Int J Radiat Oncol Biol Phys. 2016;95:1142-8.

15. Joiner M. Quantifying cell kill and survival. In: Joiner M, Van der Kogel A, editors. Basic Clinical Radiobiology. 4th ed. London: Hodder Arnold; 2009. p. 102-19.

16. Buchwald ZS, Wynne J, Nasti TH, Zhu S, Mourad WF, Yan W, Gupta S, Khleif SN, Khan MK. Radiation, Immune Checkpoint Blockade and the Abscopal Effect: A Critical Review on Timing. Dose and Fractionation Front Oncol. 2018;8:612.

17. De Maria S, Golden EB, Formenti SC. Role of Local Radiation Therapy in Cancer Immunotherapy. JAMA Oncol. 2015;1:1325-32.

18. Hodi FS, Hwu WJ, Kefford R, et al. Evaluation of Immune-Related Response Criteria and RECIST v1.1 in Patients With Advanced Melanoma Treated With Pembrolizumab. J Clin Oncol. 2016:34:1510-7.

19. Eisenhauer EA, Therasse $P$, Bogaerts J, et al. New response evaluation criteria in solid tumours: revised RECIST guideline (version 1.1). Eur J Cancer. 2009; 45:228-47.

20. Cicone F, Minniti G, Romano A, et al. Accuracy of F-DOPA PET and perfusion-MRI for differentiating radionecrotic from progressive brain metastases after radiosurgery. Eur J Nucl Med Mol Imaging. 2015;42:103-11.

21. Sperduto PW, Kased N, Roberge D, et al. Summary report on the graded prognostic assessment: an accurate and facile diagnosis-specific tool to estimate survival for patients with brain metastases. J Clin Oncol. 2012;30: 419-25

22. Knisely JP, Yu JB, Flanigan J, et al. Radiosurgery for melanoma brain metastases in the ipilimumab era and the possibility of longer survival. J Neurosurg. 2016:117:227-33.

23. Kiess AP, Wolchok JD, Barker CA, et al. Stereotactic radiosurgery for melanoma brain metastases in patients receiving ipilimumab: safety profile and efficacy of combined treatment. Int J Radiat Oncol Biol Phys. 2015;92:368-75.

24. Ahmed KA, Stallworth DG, Kim Y, et al. Clinical outcomes of melanoma brain metastases treated with stereotactic radiation and anti-PD-1 therapy. Ann Oncol. 2016:27:434-41.

25. Qian JM, Yu JB, Kluger HM, Chiang VL. Timing and type of immune checkpoint therapy affect the early radiographic response of melanoma brain metastases to stereotactic radiosurgery. Cancer. 2016;122:3051-8.

26. Choong ES, Lo S, Drummond $M$, et al. Survival of patients with melanoma brain metastasis treated with stereotactic radiosurgery and active systemic drug therapies. Eur J Cancer. 2017;75:169-78.

27. Cohen-Inbar O, Shih HH, Xu Z, Schlesinger D, Sheehan JP. The effect of timing of stereotactic radiosurgery treatment of melanoma brain metastases treated with ipilimumab. J Neurosurg. 2017:127:1007-14

28. Gaudi-Marqueste C, Dussouil AS, Carron R, et al. Survival of melanoma patients treated with targeted therapy and immunotherapy after systematic upfront control of brain metastases by radiosurgery. Eur J Cancer. 2017;84:44-54.

29. Patel KR, Shoukat S, Oliver DE, et al. I pilimumab and Stereotactic Radiosurgery Versus Stereotactic Radiosurgery Alone for Newly Diagnosed Melanoma Brain Metastases. Am J Clin Oncol. 2017:40:444-50.

30. Skrepnik T, Sundararajan S, Cui H, Stea B. Improved time to disease progression in the brain in patients with melanoma brain metastases treated with concurrent delivery of radiosurgery and ipilimumab. Oncoimmunology. 2017;6(3).

31. Chen L, Douglass J, Kleinberg L, et al. Concurrent Immune Checkpoint Inhibitors and Stereotactic Radiosurgery for Brain Metastases in Non-Small Cell Lung Cancer, Melanoma, and Renal Cell Carcinoma. Int J Radiat Oncol Biol Phys. 2018;100:916-25

32. Nardin C, Mateus C, Texier M, et al. Tolerance and outcomes of stereotactic radiosurgery combined with anti-programmed cell death-1 (pembrolizumab) for melanoma brain metastases. Melanoma Res. 2018:28:111-9.

33. Anderson ES, Postow MA, Wolchok JD, et al. Melanoma brain metastases treated with stereotactic radiosurgery and concurrent pembrolizumab display marked regression; efficacy and safety of combined treatment. J Immunother Cancer. 2017:5:76.

34. Mathew M, Tam M, Ott PA, et al. Ipilimumab in melanoma with limited brain metastases treated with stereotactic radiosurgery. Melanoma Res. 2013:23:191-5. 
35. Dovedi SJ, Cheadle EJ, Popple AL, et al. Fractionated Radiation Therapy Stimulates Antitumor Immunity Mediated by Both Resident and Infiltrating Polyclonal T-cell Populations when Combined with PD-1 Blockade. Clin Cancer Res. 2017:23:5514-26.

36. Liang H, Deng L, Chmura S, et al. Radiation-induced equilibrium is a balance between tumor cell proliferation and T cell-mediated killing. J Immunol. 2013;190:5874-81.

37. Sharabi AB, Nirschl CJ, Kochel CM, et al. Stereotactic Radiation Therapy Augments Antigen-Specific PD-1-Mediated Antitumor Immune Responses via Cross-Presentation of Tumor Antigen. Cancer Immunol Res. 2015;3:345-55.

38. Demaria S, Kawashima N, Yang AM, et al. Immune-mediated inhibition of metastases following treatment with local radiation and CTLA-4 blockade in a mouse model of breast cancer. Clin Cancer Res. 2005;11:728-34.

39. Dewan MZ, Galloway AE, Kawashima N, et al. Fractionated but not singledose radiotherapy induces an immune-mediated abscopal effect when combined with anti-CTLA-4 antibody. Clin Cancer Res. 2009;15:5379-88.

40. Vanpouille-Box C, Alard A, Aryankalayil MJ, et al. DNA exonuclease Trex1 regulates radiotherapy-induced tumour immunogenicity. Nat Commun. 2017:8:15618.

41. Postow MA, Callahan MK, Barker CA, et al. Immunologic correlates of the abscopal effect in a patient with melanoma. N Engl J Med. 2012;366:925-31.

42. Chandra RA, Wilhite TJ, Balboni TA, et al. A systematic evaluation of abscopal responses following radiotherapy in patients with metastatic melanoma treated with ipilimumab. Oncoimmunology. 2015;4:e1046028.

43. Golden EB, Chhabra A, Chachoua A, et al. Local radiotherapy and granulocyte-macrophage colony-stimulating factor to generate abscopal responses in patients with metastatic solid tumours: a proof-of-principle trial. Lancet Oncol. 2015;16:795-803.

44. Twyman-Saint Victor C, Rech AJ, Maity A, et al. Radiation and dual checkpoint blockade activate non-redundant immune mechanisms in cancer. Nature. 2015;520:373-7.

45. Kang J, Demaria S, Formenti S. Current clinical trials testing the combination of immunotherapy with radiotherapy. J Immunother Cancer. 2016;4:51.

46. Parakh S, Park JJ, Mendis S, et al. Efficacy of anti-PD-1 therapy in patients with melanoma brain metastases. Br J Cancer. 2017:116:1558-63.

47. Blonigen BJ, Steinmetz RD, Levin L, et al. Irradiated volume as a predictor of brain radionecrosis after linear accelerator stereotactic radiosurgery. Int J Radiat Oncol Biol Phys. 2010;77:996-1001.

48. Minniti G, Clarke E, Lanzetta G, et al. Stereotactic radiosurgery for brain metastases: analysis of outcome and risk of brain radionecrosis. Radiat Oncol. 2011;15(6):48

Ready to submit your research? Choose BMC and benefit from:

- fast, convenient online submission

- thorough peer review by experienced researchers in your field

- rapid publication on acceptance

- support for research data, including large and complex data types

- gold Open Access which fosters wider collaboration and increased citations

- maximum visibility for your research: over $100 \mathrm{M}$ website views per year

At $\mathrm{BMC}$, research is always in progress.

Learn more biomedcentral.com/submissions 